\title{
Association of Adventitial Vasa Vasorum and Inflammation With Coronary Hyperconstriction After Drug-Eluting Stent Implantation in Pigs In Vivo
}

\author{
Kensuke Nishimiya, MD, PhD; Yasuharu Matsumoto, MD, PhD; Tomohiko Shindo, MD; \\ Kenichiro Hanawa, MD, PhD; Yuhi Hasebe, MD, PhD; Ryuji Tsuburaya, MD, PhD; \\ Takashi Shiroto, MD, PhD; Jun Takahashi, MD, PhD; Kenta Ito, MD, PhD; \\ Hatsue Ishibashi-Ueda, MD, PhD; Satoshi Yasuda, MD, PhD; Hiroaki Shimokawa, MD, PhD
}

\begin{abstract}
Background: The importance of adventitial inflammation has been implicated for the pathogenesis of coronary artery disease. However, the roles of adventitial changes in drug-eluting stent (DES)-induced coronary hyperconstriction remain largely unknown. In the present study, this issue in pigs in vivo with a special reference to adventitial vasa vasorum (VV) formation and Rho-kinase activation, a central mechanism of coronary vasospasm, was examined.
\end{abstract}

Methods and Results: Each animal received a sirolimus-eluting stent (SES) and a biolimus A9-eluting stent (BES), one in the left anterior descending and another in the left circumflex coronary arteries in a randomized manner $(n=18)$. After 1,3 and 6 months, coronary vasomotion was examined. At 1 month, coronary vasoconstriction to serotonin was significantly enhanced at the SES edges as compared with the BES edges (SES, $52 \pm 7 \%$ vs. BES, $22 \pm 3 \%, P<0.01$ ), which was equally prevented by a selective Rho-kinase inhibitor, hydroxyfasudil. A significant difference in vasoconstriction between SES and BES was sustained for 6 months. A micro-CT showed VV augmentation at the SES site, extending to the proximal and distal edges. Immunostainings demonstrated that VV formation, macrophage infiltration in the adventitia and Rho-kinase expressions/activation were significantly enhanced at the SES edges as compared with the BES edges.

Conclusions: The DES with durable polymers enhances VV formation and inflammation in the adventitia, associating with the pathogenesis of DES-induced coronary hyperconstriction through Rho-kinase activation in pigs in vivo. (Circ J 2015; 79: 1787-1798)

Key Words: Adventitia; Rho-kinase; Spasm; Stents; Vasoconstriction

$\mathbf{T}$ he widespread utilization of drug-eluting stents (DES) has remarkably reduced the risk of re-stenosis and of repeated revascularization. ${ }^{1}$ However, the first-generation DES with durable polymers, such as sirolimus-eluting stents (SES), have no beneficial prognostic effects as compared with bare metal stents ${ }^{2}$ or even cause unfavorable effects, such as stent thrombosis ${ }^{3,4}$ and coronary hyperconstricting responses, ${ }^{5-8}$ not only to acetylcholine, ${ }^{5}$ but also to exercise ${ }^{6}$ and atrial pacing. ${ }^{7}$

\section{Editorial $p 1693$}

In order to reduce the adverse effects of the first-generation DES, many efforts have been made, including the development of a newer biocompatible DES. ${ }^{9}$ Biolimus-A9 eluting stent (BES) is one of the new-generation DES with unique characteristics, such as biodegradable polymers that are absorbed into the arterial wall approximately 6 months after stent implantation. ${ }^{9-11}$ Although BES has recently been shown to preserve ex-vivo coronary vasomotion as compared with SES in the short-term observations in pigs ${ }^{12}$ and humans, ${ }^{7}$ its mechanism remains largely unknown.

We have previously demonstrated that Rho-kinase activation is a central molecular mechanism of coronary spasm in

Received February 6, 2015; revised manuscript received April 13, 2015; accepted April 29, 2015; released online June 1, 2015 Time for primary review: 26 days

Department of Cardiovascular Medicine, Tohoku University Graduate School of Medicine, Sendai (K.N., Y.M., T. Shindo, K.H., Y.H., R.T., T. Shiroto, J.T., K.I., H.S.); Department of Pathology (H.I.-U.), Department of Cardiovascular Medicine (S.Y.), National Cerebral and Cardiovascular Center, Suita, Japan

The Guest Editor for this article was Masakazu Yamagishi, MD.

Mailing address: Hiroaki Shimokawa, MD, PhD, Department of Cardiovascular Medicine, Tohoku University Graduate School of Medicine, Seiryo-machi, Aoba-ku, Sendai 980-8574, Japan. E-mail: shimo@cardio.med.tohoku.ac.jp

ISSN-1346-9843 doi:10.1253/circj.CJ-15-0149

All rights are reserved to the Japanese Circulation Society. For permissions, please e-mail: cj@j-circ.or.jp 


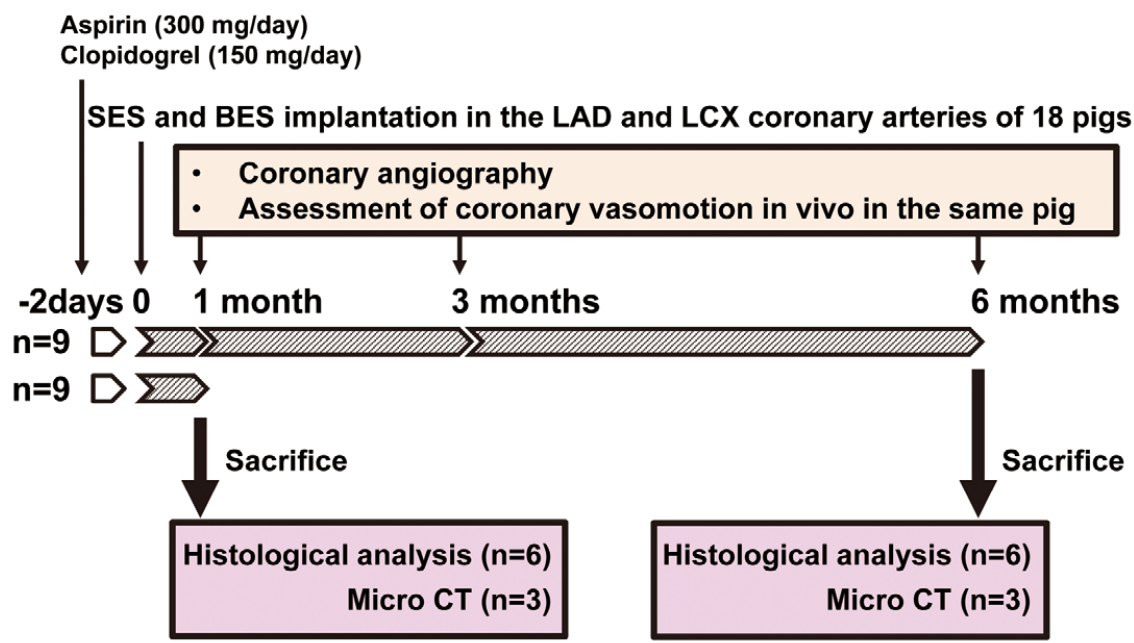

Figure 1. Study protocol. A sirolimus-eluting stent (SES) and a biolimus A9-eluting stent (BES) were randomly implanted into the left anterior descending (LAD) and the left circumflex (LCX) coronary arteries in the same miniature pig $(n=18$ each). At 1 month after stent implantation, all animals underwent follow-up coronary angiography for the assessment of coronary vasomotion, and 9 of them were euthanized for histological analysis ( $n=6$ each) and micro-computed tomography (CT) ( $n=3$ each). The remaining 9 animals were kept for 6 months and again underwent coronary angiography for the assessment of coronary vasomotion at 3 and 6 months ( $n=9$ each). After an in vivo experiment at 6 months, they were euthanized for histological analysis ( $n=6$ each) and microCT $(n=3$ each).

animals and humans, ${ }^{8,13,14}$ and that it also plays a key role in the pathogenesis of coronary hyperconstricting responses after the first-generation DES implantation in animals ${ }^{15,16}$ and humans ${ }^{5}$ as compared with the bare-metal stents. Nevertheless, it remains to be elucidated why DES-induced coronary hyperconstriction spontaneously develops at the proximal and distal stent edges. We and others have previously demonstrated the importance of adventitial inflammatory changes in the pathogenesis of coronary spasm in pigs and humans. . $^{8,13,14,17,18}$ Enhanced vasa vasorum (VV) formation that leads to the adventitial inflammation has been shown to contribute to atherosclerotic plaque progression ${ }^{19-22}$ and vascular healing after balloon injury in pigs. ${ }^{23}$ However, it also remains to be fully elucidated whether adventitial inflammation, including VV formation as a conduit, plays a role in the pathogenesis of DES-induced coronary hyperconstriction.

In the present study, we thus examined whether BES inhibits coronary hyperconstricting responses as compared with SES for up to 6 months in pigs in vivo, and if so, what mechanisms are underlying it, with special reference to adventitial VV formation, inflammatory changes and Rho-kinase activation, particularly at the proximal and distal stent edges in pigs in vivo.

\section{Methods}

All animal care and experiments were performed in accordance with the Guide for the Care and Use of Laboratory Animals published by the U.S. National Institute of Health (NIH Publication, 8th Edition, 2011), and were approved by the Institutional Committee for Use of Laboratory Animals of Tohoku University (2013MdA-059).

\section{Animal Preparation}

Göttingen miniature pigs (male, 1 year old and weighing
$25-30 \mathrm{~kg}$ ) were pre-treated orally with aspirin $(200 \mathrm{mg} /$ day $)$ and clopidogrel ( $225 \mathrm{mg} /$ day) for 2 days before stent implantation. After sedation with medetomidine $(0.1 \mathrm{mg} / \mathrm{kg}, \mathrm{IM})$ and midazolam $(0.2 \mathrm{mg} / \mathrm{kg}, \mathrm{IM})$, followed by inhaled sevoflurane (2-5\%) and heparinization (5,000 U, IV), each animal received a SES (Cypher Select; Johnson \& Johnson, New Brunswick, NJ, USA) and a BES (Nobori; Terumo Corporation, Tokyo, Japan). One stent was implanted into the left anterior descending coronary artery and another stent into the left circumflex coronary artery in the same pig in a randomized manner $(n=18)$ (Figure 1). The balloon inflation ratio was adjusted to achieve an overstretch ratio of 1.0-1.1 under the guidance of intravascular ultrasound (View IT; Terumo Corporation, Tokyo, Japan). There was no difference in the stent implantation procedure between the 2 sites (Table S1). The anti-platelet therapy with orally given aspirin (100 mg/day) and clopidogrel ( $75 \mathrm{mg} /$ day) was continued until euthanasia.

\section{In Vivo Assessment of Coronary Vasomotion}

At 1, 3 and 6 months after stent implantation, we repeated the coronary angiography to examine coronary vasomotion in the same pigs in vivo (Figure 1). Coronary vasoconstricting responses to serotonin (10 and $100 \mu \mathrm{g} / \mathrm{kg}$, IC) were examined before and after hydroxyfasudil administration (30 and $100 \mu \mathrm{g} / \mathrm{kg}$, IC for $3 \mathrm{~min}$ ), a specific Rho-kinase inhibitor (Y27632; Asahi Kasei Pharma, Tokyo, Japan). ${ }^{8,14}$ Coronary vasodilating responses to nitroglycerin $(10 \mu \mathrm{g} / \mathrm{kg}$, IC) and bradykinin $(0.1 \mu \mathrm{g} / \mathrm{kg}, \mathrm{IC})$ before and after $\mathrm{N}^{\mathrm{G}}$-monomethyl-Larginine administration (L-NMMA) (1 mg/kg, IC for $10 \mathrm{~min}$ ) were examined. ${ }^{15,16}$ Quantitative coronary angiography (QCA) (INFX-8000V; Toshiba Medical Co, Tokyo, Japan) was performed in a blind manner at the proximal and distal stent edge segments adjacent to the stent $(5 \mathrm{~mm}$ apart from the proximal and distal stent end portions), as previously described. ${ }^{5}$ 


\section{Histological Analysis}

At 1 and 6 months after stent implantation, the animals were euthanized with a lethal dose of potassium chloride $(0.25 \mathrm{mEq} / \mathrm{kg}$, IV) after deep sedation with a high concentration of inhaled $5 \%$ sevoflurane; the heart was then removed ( $\mathrm{n}=6$, each) (Figure 1). The saline and 10\% neutral buffered formalin were infused into the left coronary arteries via a constant perfusion pressure system $\left(120 \mathrm{~cm} \mathrm{H}_{2} \mathrm{O}\right){ }^{15,16}$ After the fixation, the stented vessels were isolated for histological analysis. Vessels were separated into the stent implantation sites for the resin sections and the stent edges ( $5 \mathrm{~mm}$ apart from the stent) for the paraffin sections. The resin sections of stent implantation sites were divided in the proximal, middle and distal segments, and were cut into a 3-5 $\mu \mathrm{m}$-thick slices. The paraffin sections of stent edge sites were cut into a $3 \mu \mathrm{m}$-thick slices. All sections were stained with hematoxylin-eosin, Elastica van Gieson, Masson's trichrome and toluidine blue stainings. Histomorphometric parameters were manually measured by using Image-J (U.S. National Institute of Health, Bethesda, MD, USA). The adventitial area was calculated by using the following formula: [Area outside external elastic lamina (EEL) within a distance of the thickness of neointima plus mediaEEL area], as previously described. ${ }^{22,23}$ The ratio of endothelialization was calculated as the stent struts covered with endothelium per total struts. ${ }^{3}$ The injury score for the stent implantation site was evaluated by using the following scale: $0=$ internal elastic lamina intact; $1=$ internal elastic lamina lacerated; $2=$ internal elastic lacerated; and $3=E E L$ lacerated. ${ }^{24}$ The extent of inflammatory changes around each stent strut per 1 section was assessed by using the following scale: $0=$ none; $1=$ fewer than 5 inflammatory cells; $2=$ fewer than 20 inflammatory cells; and $3=$ more than 20 inflammatory cells, as previously described. ${ }^{15,16}$ The extent of microthrombus formation around each stent strut was assessed by using the following scale: $0=$ none; $1=$ minute thrombus persistence; $2=$ thrombus $<50 \%$ circular area of persistence; and $3=$ thrombus all around stent strut, as previously described. ${ }^{15,16}$

\section{Immunohistological Analysis}

Immunoreactivities were examined, by using rabbit antihuman von Willebrand factor (vWF) antibody (1:3) (N1505; Dako, Copenhagen, Denmark) for VV, mouse anti-human vascular endothelial growth factor (VEGF) antibody (1:50) (VG76e; Novus Biologicals, Littleton, CO, USA), rabbit anti-human CD11c antibody (1:50) (NB11055521; Novus Biologicals) for pro-inflammatory M1-macrophages, ${ }^{25}$ rabbit anti-mannose receptor (CD206) antibody (1:5,000) (ab64693; Abcam, Cambridge, UK) for anti-inflammatory M2-macrophages, ${ }^{25}$ and goat anti-human interleukin (IL)-1 $\beta$ (1:50) (AB201-NA; R\&D Systems, Minneapolis, MN, USA). The density of VV, M1- and M2-macrophages was expressed as the number of vWF, CD11c and CD206 positive-cells divided by the adventitial area $\left(\mathrm{mm}^{2}\right)$. Immunohistochemical staining was also performed by using mouse anti-human Rho-kinase $\beta$ (ROCK1) (1:50) (611136; BD Biosciences, San Jose, CA, USA) and Rho-kinase $\alpha$ (ROCK2) antibodies (1:50) (610624; BD Biosciences), rabbit anti-human phosphorylated myosin phosphatase target subunit-1 (pMYPT1) antibody (1:50) (07251; Millipore, Billerica, MA, USA), a substrate of Rhokinase,, 14 and appropriate non-immune immunoglobulin (IgGs) in each case. Each section was divided into a total of 12 radial subparts. Semi-quantitative analysis regarding the extent of VEGF, ROCK1, ROCK2 and pMYPT1 was evaluated for each radial subpart per 1 section by using the following scale: $0=$ none; $1=$ slight; $2=$ moderate; and $3=$ high, as previously described. ${ }^{15,16}$

\section{Micro-Computed Tomography}

At 1 and 6 months after stent implantation, the heart was removed after euthanization for micro-computed tomography (micro-CT) analysis ( $\mathrm{n}=3$ each) (Figure 1). Heparinized saline was infused into the left coronary arteries via a constant perfusion pressure system $\left(120 \mathrm{~cm} \mathrm{H}_{2} \mathrm{O}\right){ }^{15,16}$ The left coronary arteries were subsequently perfused with a silicone rubber compound (MV122; Flow Tech, Carver, MA, USA) and fixed with $10 \%$ neutral buffered formalin for $48 \mathrm{~h} .{ }^{19,21,23}$ The isolated coronary arteries were then emerged with $25-100 \%$ concentrationraised ethanol for 7 days and then with methyl salicylate for $24 \mathrm{~h}$ and were left in open air for $24 \mathrm{~h}$. The specimens were scanned by a micro-CT system (24-48 $\mu \mathrm{m}$ voxel size) (LCT200; Hitachi-Aloka, Tokyo, Japan). 3D micro-CT images were reconstructed by VG Studio MAX (Volume Graphics GmbH, Heidelberg, Germany)

\section{Statistical Analysis}

Results are expressed as mean \pm standard error of mean (SEM). In the QCA analysis, $\mathrm{n}$ represents the number of the stent edges including the proximal and distal sites. Throughout the text and figures on the histology, $\mathrm{n}$ represents the number of sections. A comparison of the QCA and histomorphometry was performed by using an unpaired, 2-sided Student's t-test. Comparison of the semi-quantitative analysis was performed by using a Mann-Whitney U-test. The correlation between the continuous variables was analyzed using a linear regression model. Statistical analysis was performed with IBM SPSS Statistics 20 (IBM, New York, NY, USA). A value of $\mathrm{P}<0.05$ was considered to be statistically significant.

\section{Results}

In the 18 miniature pigs examined, SES and BES were alternately implanted into the left anterior descending and left circumflex coronary artery. There was no significant difference in the stent implantation procedure between the 2 groups (Table S1).

\section{Coronary Vasomotion After Stent Implantation}

At 1 month after stent implantation, there was no in-stent restenosis at the 2 stent implantation sites (Figure 2A). Coronary hyperconstricting responses to intracoronary serotonin were noted at the proximal and the distal edges of the SES sites, which were markedly attenuated at the BES edges (Figure 2B), and both of them were abolished by pre-treatment with intracoronary hydroxyfasudil, a selective Rho-kinase inhibitor (Figure 2C). Coronary angiography and quantitative coronary angiography showed that the difference in serotonininduced coronary vasoconstricting responses between the SES and BES edges persisted throughout the experimental period until 6 months (Figures 2D-I), whereas endothelium-independent and -dependent coronary vasodilating responses to nitroglycerin and bradykinin (regardless of the presence or absence of $\mathrm{N}^{\mathrm{G}}$-monomethyl-L-arginine), respectively, were comparable between the SES and BES edges throughout the experimental periods (Figure $\mathbf{S 1}$ ).

\section{Histomorphological Analysis at the Stent Implantation Sites and the Stent Edges}

At 1 and 6 months after stent implantation, histomorphometric parameters at the stent implantation sites (Table S2 and Figures S2A-L) and those at the stent edges (Table S3) were 


\section{1 month}

\section{A Nitroglycerin B Serotonin}
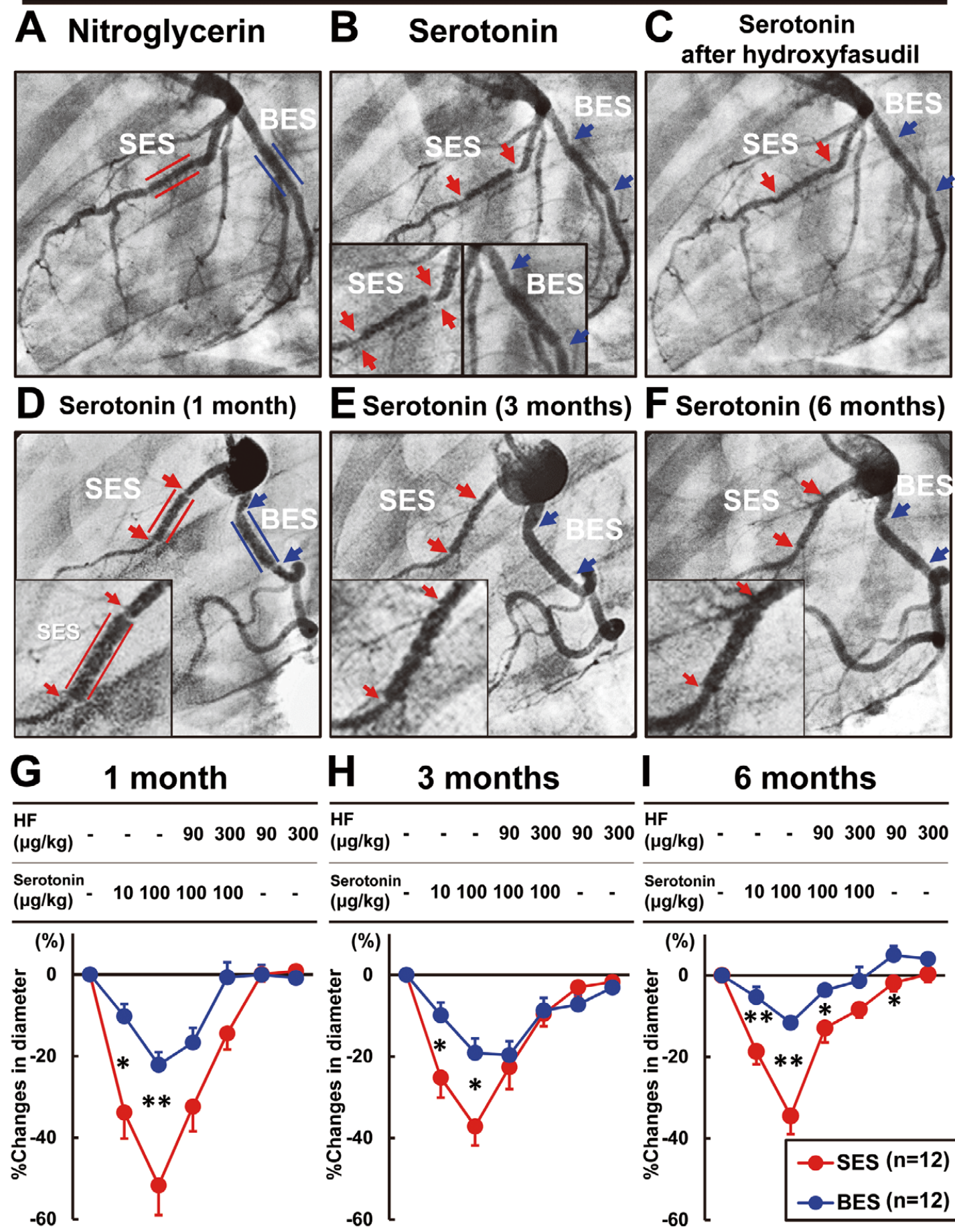

$\begin{aligned} & \text { Serotonin } \\ & (\mu \mathrm{g} / \mathrm{kg})\end{aligned} 10100100100--$
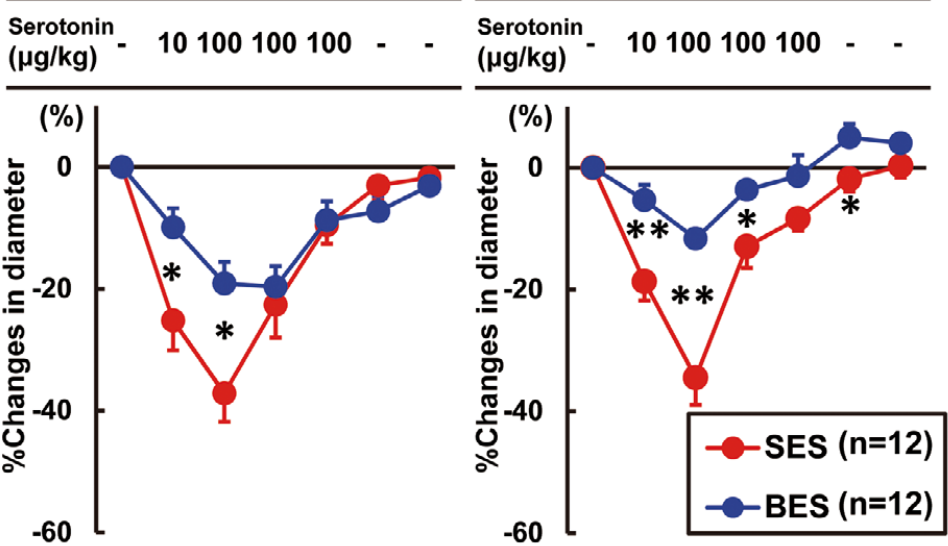

Figure 2. Coronary vasoconstricting responses at the sirolimus-eluting stent (SES) and the biolimus A9-eluting stent (BES) edges after stent implantation in pigs in vivo. Representative left coronary angiograms at 1 month after the administration of nitroglycerin $(10 \mu \mathrm{g} / \mathrm{kg}, \mathrm{IC})(\mathbf{A})$, serotonin $(100 \mu \mathrm{g} / \mathrm{kg}, \mathrm{IC})$ alone (B), and serotonin after pre-treatment with hydroxyfasudil $(300 \mu \mathrm{g} / \mathrm{kg} \mathrm{IC})(\mathbf{C})$. Time-course of coronary hyperconstricting responses shown by representative left coronary angiograms of the same animal after intracoronary serotonin injection $(100 \mu \mathrm{g} / \mathrm{kg}, \mathrm{IC})$ at 1 (D), $3(\mathrm{E})$, and 6 (F) months. The red and blue lines represent a SES in the left anterior descending (LAD) and a BES in the left circumflex (LCX) coronary arteries, respectively (A,D). The red and blue arrows represent the stent edges of the SES and the BES sites, respectively $(\mathbf{A}-\mathbf{F})$. Results are presented from a quantitative coronary angiography regarding coronary vasoconstricting responses to serotonin (10 and $100 \mu \mathrm{g} / \mathrm{kg}$, IC) before and after hydroxyfasudil (HF) $(90$ and $300 \mu \mathrm{g} / \mathrm{kg}, \mathrm{IC}$ ) at 1 (G), $3(\mathrm{H})$ and 6 (I) months. The mean value of coronary vasoconstricting responses of the proxi$\mathrm{mal}$ and the distal stent edges are presented. Results are expressed as mean $\pm \mathrm{SEM}$. ${ }^{*} \mathrm{P}<0.05,{ }^{*} \mathrm{P}<0.01 \mathrm{SES}$ vs. BES. 


\section{1 month}

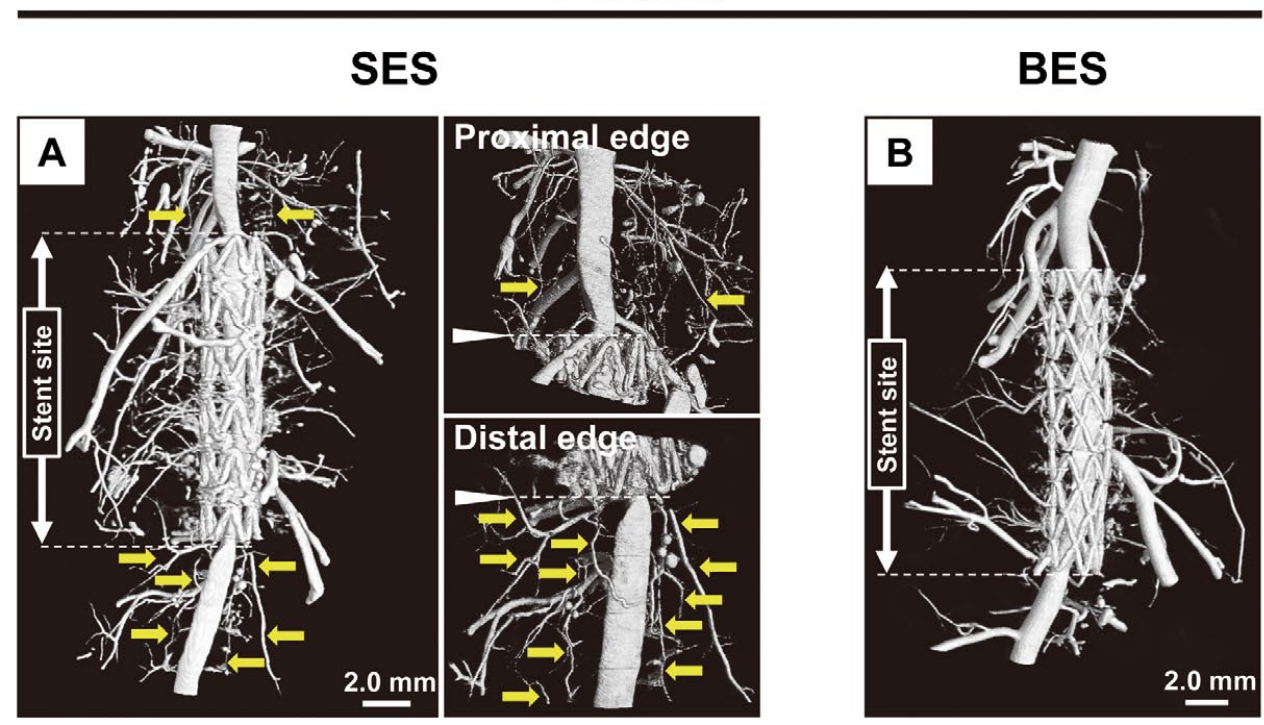

\section{6 months}

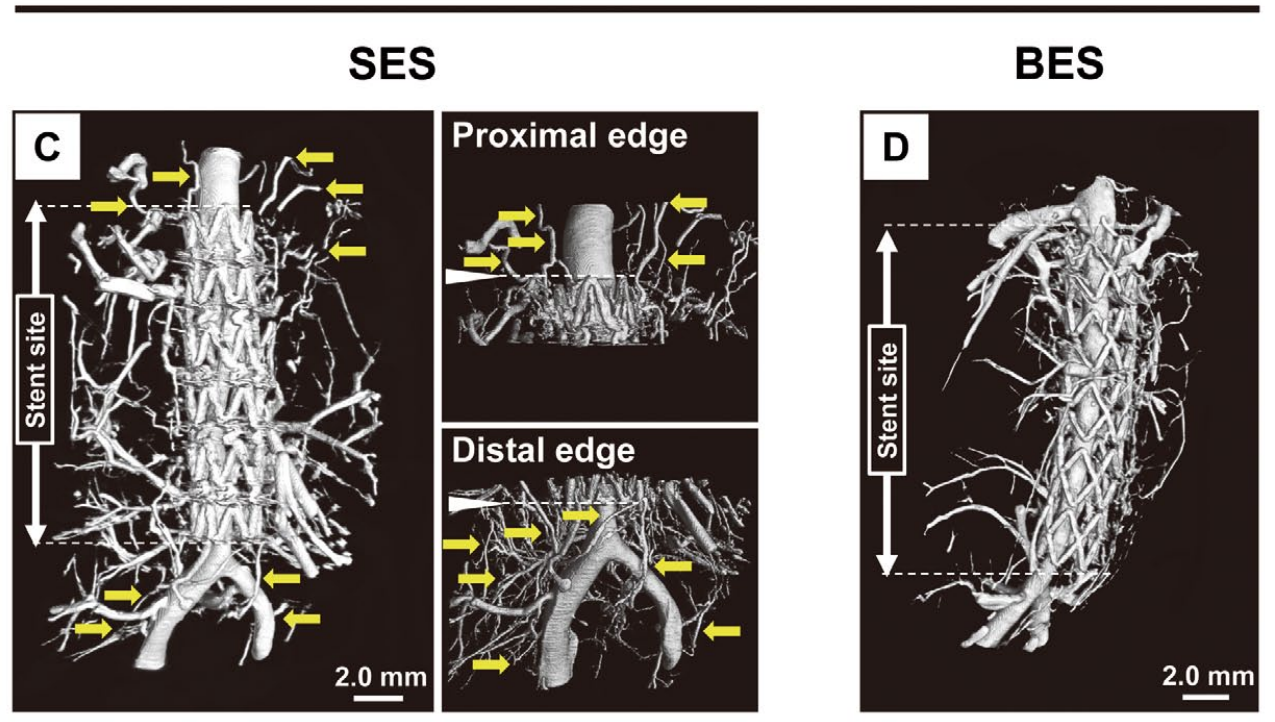

Figure 3. Vasa vasorum (VV) formation in the sirolimus-eluting stent (SES) and the biolimus A9-eluting stent (BES) sites, evaluated by micro-CT imaging. Representative micro-computed (CT) images ( $48 \mathrm{~mm}$ voxel size) of the left anterior descending (LAD) coronary arteries implanted with SES at 1 (A) and 6 (C) months, and those implanted with BES at 1 (B) and 6 (D) months ( $n=3$ each). Magnified images of the proximal and distal SES edges ( $24 \mu \mathrm{m}$ voxel size) are shown at 1 and 6 months $(\mathbf{A}, \mathbf{C})$. The white two-way arrows indicate the stent implantation sites and the dashed-lines indicate the proximal and distal stent edges. The VV formation at the SES edges is shown by yellow arrows.

comparable between the SES and BES sites.

\section{Micro-CT Images}

Micro-CT images showed that adventitial VV formation was dramatically enhanced at the SES site (Figures 3A,C) as compared with the BES site (Figures 3B,D) at 1 and 6 months after stent implantation. In the magnified images, the VV network appeared to extend from the SES implantation site to the proximal and distal edge portions (Figures $\mathbf{3 A}, \mathbf{C}$ ).
Adventitial VV Formation at the Stent Implantation Sites and the Stent Edges

At the stent implantation sites, Masson's trichrome staining showed that the number of microvessels in the adventitia was significantly increased in the adventitia of the SES stent sites as compared with the BES stent sites at 1 month (BES; $20.0 \pm 2.2$ vs. SES; 55.6 $\pm 4.8, \mathrm{P}<0.01)$ and at 6 months (BES; $21.8 \pm 2.6$ vs. SES; 55.0 $\pm 7.0, \mathrm{P}<0.01$ ) (Figures $4 \mathrm{~A}-\mathrm{D}$ ). Similarly, at the stent edge portions, those adventitial microvessels were 

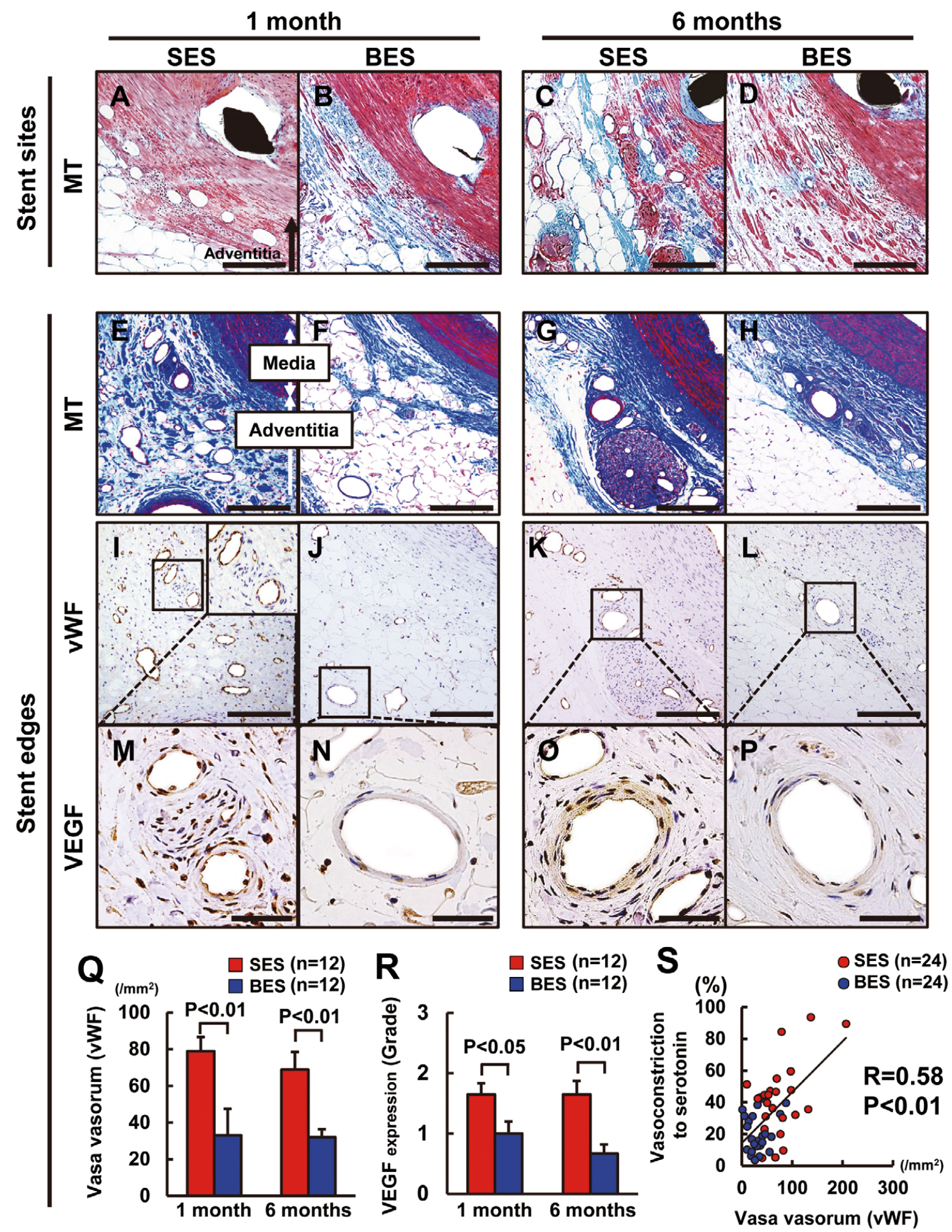

Figure 4. Adventitial vasa vasorum (VV) after stent implantation. Representative pictures of the sirolimus-eluting stent (SES) and biolimus A9-eluting stent (BES) stent implantation sites at 1 and 6 months for Masson-trichrome stainings (MT) (A-D), the SES and BES edges at 1 and 6 months for MT (E-H), von Willebrand factor (VWF) (I-L), and vascular endothelial growth factor (VEGF) stainings (M-P). Scale bars represent $200 \mu \mathrm{m}(\mathbf{A}-\mathbf{L})$ and $50 \mu \mathrm{m}(\mathbf{M}-\mathbf{P})$. A right upper panel shows the magnified image of vWF staining (I). Quantitative/semiquantitative analysis at 1 and 6 months of VV density (Q) and VEGF expression (R). Correlations between the VV density and the extent of serotonin $(100 \mu \mathrm{g} / \mathrm{kg}, \mathrm{IC})$-induced coronary vasoconstricting responses at the stent edges at 1 and 6 months $(\mathbf{S})$. Results are expressed as mean \pm SEM. 


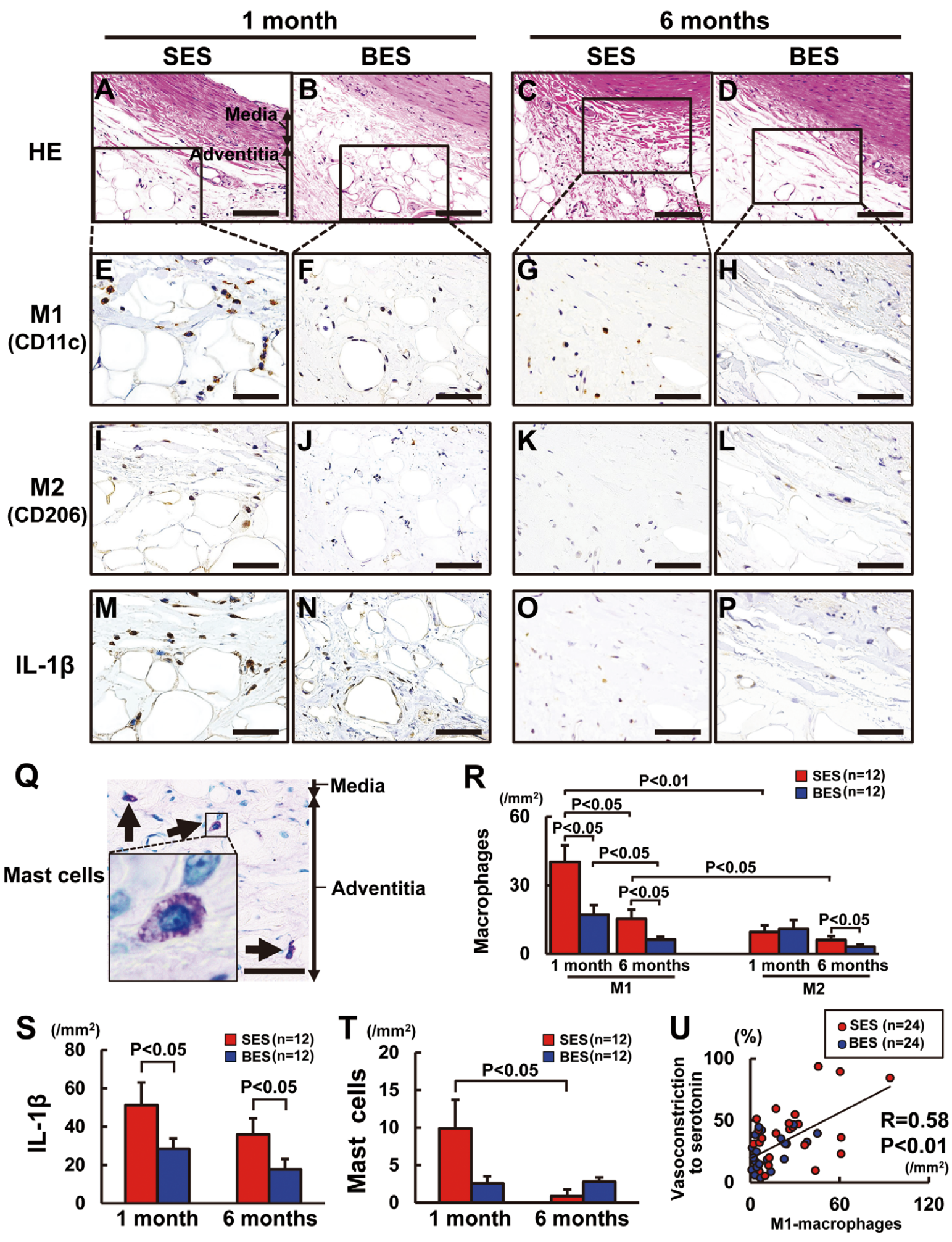

Figure 5. Adventitial inflammatory changes at the stent edge sites. Representative pictures of the sirolimus-eluting stent (SES) and biolimus A9-eluting stent (BES) edges at 1 and 6 months for hematoxylin-eosin (HE) stainings (A-D), immunostainings for M1 (CD11c-positive) (E-H) and M2 (CD206-positive) macrophages (I-L), and interleukin (IL)-1 $\beta$ (M-P). Toluidine blue-positive mast cells at the SES edge at 1 month (Q). Scale bars represent $100 \mu \mathrm{m}(\mathbf{A}-\mathbf{D})$ and $50 \mu \mathrm{m}(\mathbf{E}-\mathbf{Q})$. Quantitative analysis of cell number in the adventitia at 1 and 6 months for macrophages (R), IL-1 $\beta$-positive cells (S) and mast cells (T). Correlations between M1-macrophage density and the extent of serotonin $(100 \mu \mathrm{g} / \mathrm{kg}$, IC)-induced coronary vasoconstricting responses at the stent edges at 1 and 6 months (U). Results are expressed as mean \pm SEM. 

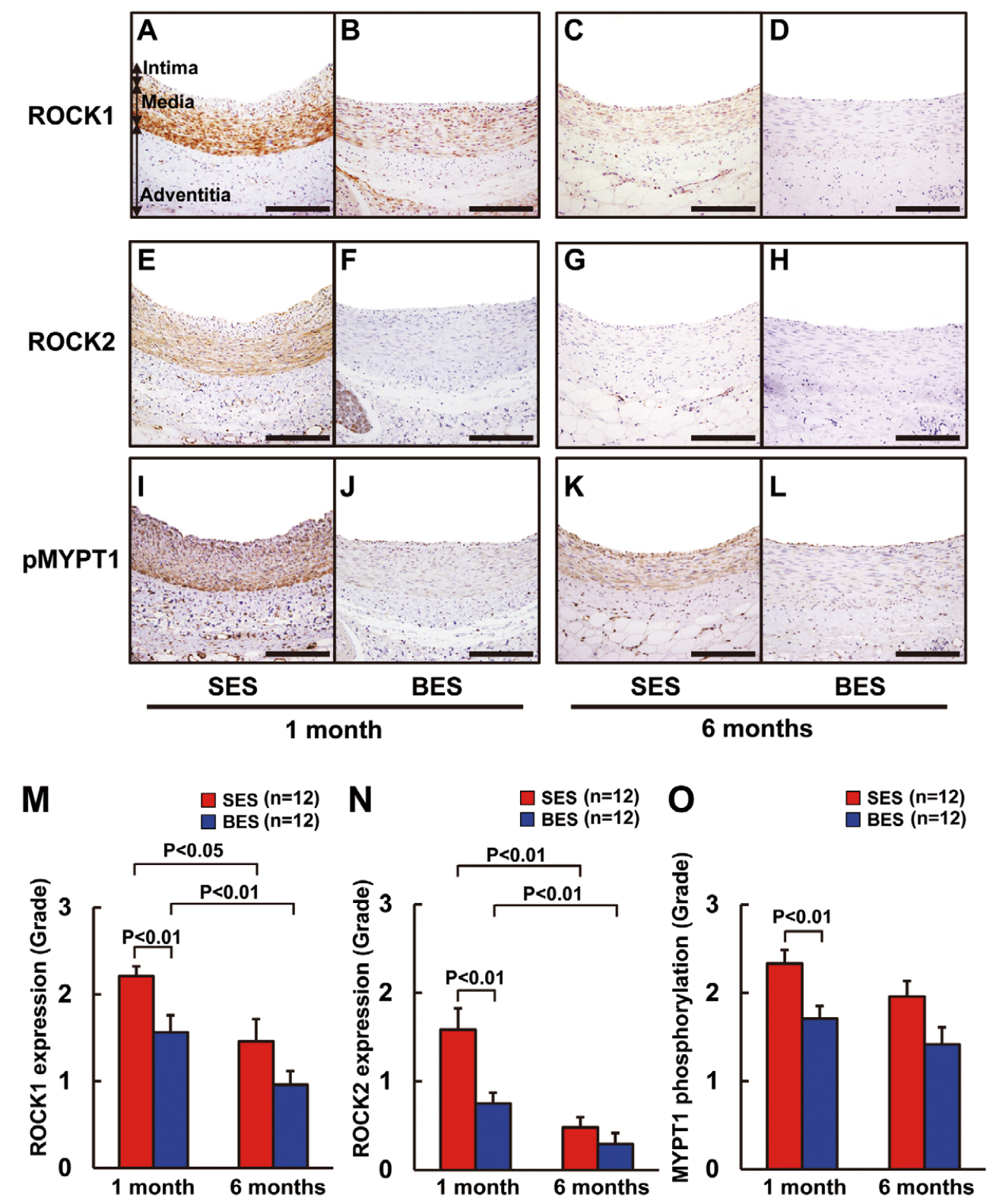

Figure 6. Immunohistological analysis of Rho-kinase expression and activity at the stent edge sites. Representative immunostainings of the sirolimus-eluting stent (SES) and biolimus A9-eluting stent (BES) edges at 1 and 6 months for ROCK1 (Rho-kinase $\beta$ ) (A-D), ROCK2 (Rho-kinase a) (E-H), and phosphorylated myosin phosphatase target subunit-1 (pMYPT1) (a marker of Rho-kinase activation) (I-L). Scale bars represent $200 \mu \mathrm{m}$. Results of semi-quantitative analysis for the extent of ROCK1 (M), ROCK2 (N) and pMYPT1 (0). Results are expressed as mean \pm SEM.

predominantly noted in the SES edges as compared with the BES edges (Figures $4 \mathrm{E}-\mathrm{H}$ ), which were VV as they were positive for vWF staining (Figures 4I-L). The VV density was significantly increased at the SES edges as compared with the BES edges at 1 and 6 months (Figure 4Q). The expression of VEGF was noted predominantly around VV at the SES edges as compared with the BES edges (Figures $4 \mathrm{M}-\mathrm{P}$ ). Notably, $\mathrm{VV}$ at the SES edges was also positive for VEGF-R staining (Figure S3E). VEGF appeared to be predominately expressed in both pericytes of VV and adventitial inflammatory cells, whereas VEGF-R was highly expressed in pericytes of VV (Figure S3). Furthermore, semi-quantitative analysis demonstrated that the extent of VEGF expression was significantly greater at the SES edges compared with the BES edges at 1 and 6 months (Figure 4R). Notably, there was a significant positive correlation between the adventitial VV density at the stent edge vasoconstriction sites and the extent of serotonininduced coronary vasoconstriction (Figure 4S). 


\section{Adventitial Inflammatory Changes at the Stent Implantation Sites and the Stent Edges}

At the stent implantation sites, the extent of inflammatory changes and that of microthrombus formation were significantly increased at the SES edges as compared with the BES edges at 1 month, and both changes were significantly attenuated at 6 months (Figures S2I-N). Similarly, at the stent edge portions, inflammatory cells infiltration was predominantly noted in the adventitia of the SES edges as compared with the BES edges at 1 and 6 months after stent implantation (Figures 5A-D). Indeed, the numbers of pro-inflammatory CD11c-positive cells (M1-macrophages) (Figures 5E-H) and IL-1 $\beta$ positive cells (Figures 5 M-P) were significantly increased at the SES edges as compared with the BES edges, and were then diminished at 6 months in both groups (Figures 5R,S). In contrast, anti-inflammatory CD206-positive cells (M2-macrophages) were less observed in both groups (Figures $5 \mathbf{I}-\mathbf{L}, \mathbf{R})$. Toluidine blue-positive mast cells were detected more frequently at the SES edges as compared with the BES edges at 1 month (Figures 5Q,T). The mast cells density tended to be positively correlated with the extent of serotonin-induced coronary vasoconstriction $(\mathrm{P}=0.06, \mathrm{R}=0.28)$. Furthermore, there was a significant positive correlation between M1-macrophage density in the adventitia of the stent edge vasoconstriction sites and the extent of serotonin-induced coronary vasoconstriction (Figure 5U), whereas no such correlation was noted for M2-macrophages. The extent of serotonin-induced coronary vasoconstriction, that of VV formation and other inflammatory parameters at the distal edges, tended to be higher as compared with those at the proximal edges (Table S4).

\section{Rho-Kinase Activity at the Stent Edges}

Immnunoreactivities for Rho-kinase (ROCK1 and ROCK2) and pMYPT1 (a marker of Rho-kinase activity) were enhanced chiefly in the adventitial inflammatory cells and in the medial vascular smooth muscles (VSMCs) of the SES edges as compared with the BES edges at 1 month, and were then attenuated at 6 months (Figure 6). In the semi-quantitative analysis, no statistically significant differences in the extent of ROCK $1 / 2$ expressions were noted between the SES edges and the BES edges at 6 months, whereas the extent of Rho-kinase activation (pMYPT1) of the SES edges tended to be higher as compared with the BES edges $(\mathrm{P}=0.05)$ (Figures $6 \mathrm{M}-\mathrm{O}$ ). Importantly, there were significant positive correlations between the extent of Rho-kinase expressions/activity at the stent edge vasoconstriction sites and that of serotonin-induced coronary vasoconstriction (Figure S5). Moreover, there were significant positive correlations between M1-macrophage density and the extent of Rho-kinase expressions/activity (Figure S6), while no such correlation was noted for M2-macrophages (Figures S4B-D). At the SES edges, no de-differentiation of the smooth-muscle myosin heavy chain isoforms was noted (Figure S3K,L).

\section{Discussion}

The major findings of the present study were that: (1) BES, as compared with SES, significantly reduced coronary hyperconstricting responses for 6 months after stent implantation through Rho-kinase inhibition in pigs in vivo; and (2) those functional alterations of the coronary arteries at the stent edges were significantly associated with enhanced VV growth, inflammatory changes and Rho-kinase activation (Figure 7).

\section{Beneficial Effects of BES on Coronary Vasomotion}

The first-generation DES, such as SES, has already been deployed in millions of patients all over the world and has dramatically reduced the risk of the late lumen loss. ${ }^{2,9}$ In contrast, SES has fundamental limitations as they have long-term polymer residues of non-bioabsorbable nature, resulting in the delayed structural ${ }^{3,4}$ and functional healing ${ }^{4-7}$ of stented coronary segments. Impaired coronary vasomotion is associated with increased cardiovascular risks. ${ }^{26}$ Indeed, we have reported the cardiac arrest due to SES-induced coronary hyperconstriction in a patient who had stent implantation for 5 years. ${ }^{4}$ DES-induced coronary hyperconstriction is thus implicated in the long-term safety after stent implantation. In this study, we used a newer generation BES with bioabsorbable polymers coated only on the abluminal surface, which is set to be degraded in the arterial wall for up to 6 months..$^{9-12}$ Although BES has recently been shown to preserve ex-vivo porcine coronary vasomotion as compared with SES at 1 month after stent implantation, ${ }^{12}$ when more than $50 \%$ of polymers of BES still remains on the stent. ${ }^{9}$ In the present study, we were able to demonstrate for the first time that coronary hyperconstricting responses were sustained from 1 to 6 months after SES implantation, whereas BES significantly preserved coronary vasomotion over a long-term period in pigs in vivo. Those beneficial effects of BES might be provided partially by the abluminal coating and the modified biocompatibility of the polymers. Because the vascular healing process after coronary stent implantation requires approximately 6 times longer in humans than in pigs, ${ }^{27}$ the present findings may be consistent with the clinical finding that the long-term beneficial effects of BES as compared with SES are noted for up to 3 or 4 years after implantation..$^{11}$ It is thus conceivable that BES has beneficial aspects on not only vascular healing but also on coronary vasomotion, which should be a feature of the sophisticated devices for coronary intervention.

Enhanced Adventitial VV Formation After DES Implantation The structural changes in the adventitia (eg, VV formation and inflammatory changes) could be a sign of impending vascular disease. 8,13,14,17-23,28 Although a previous study showed enhanced VV formation in the rabbit aorta after SES implantation, ${ }^{29}$ the present study demonstrates for the first time that SES enhances VV formation in the porcine coronary artery not only at the stent site but also at the proximal and distal edge portions where coronary hyperconstricting responses developed. Intriguingly, it was previously demonstrated that prominent VV neovascularization in the adventitia occurs prior to the initiation of coronary lesion formation in apolipoprotein E-deficient mice $^{22}$ and hypercholesterolemic pigs. ${ }^{30}$ In this study, VEGF expression was enhanced around adventitial VV at the SES edges, indicating the occurrence of $\mathrm{VV}$ neovascularization after the SES implantation. Thus, the present study results suggest that VV formation after DES implantation is a novel therapeutic target to prevent the inflammation and the consequent coronary hyperconstriction after DES implantation. To inhibit the enhanced VV formation after DES implantation, several anti-angiogenic strategies could be proposed. First, based on the present study findings, novel biocompatible stents can be used as a promising device. Second, drug therapy with anti-angiogenic effects, such as statins, ${ }^{19}$ and plasminogen activator inhibitor- $1^{21}$ might be useful.

Adventitial Inflammatory Changes After DES Implantation In addition to VV neovascularization, the adventitia is a major 


\section{Normal coronary artery}

First-generation DES site

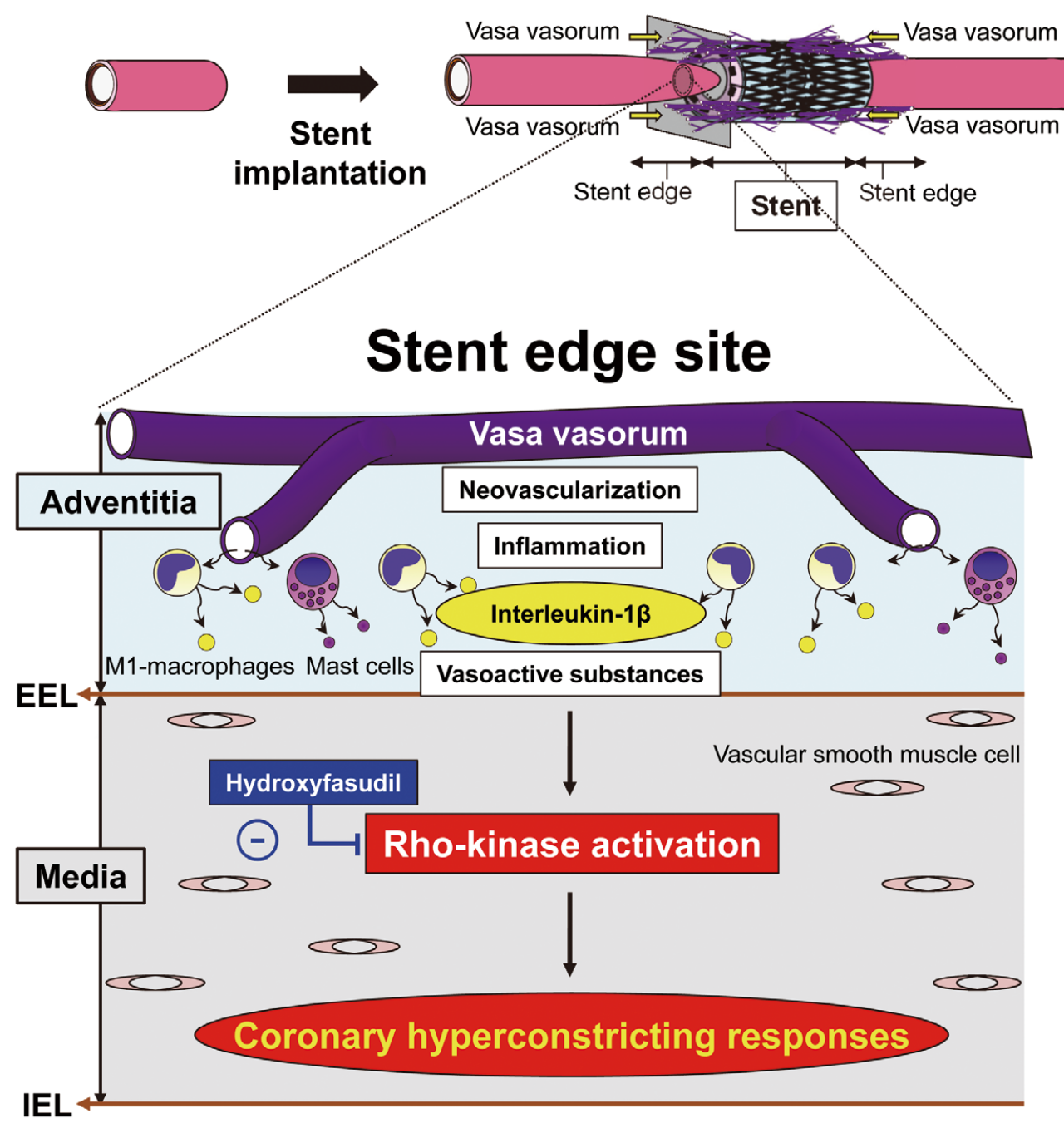

Figure 7. Summary of the present study and working hypothesis. After implantation of the first-generation DES into the coronary artery, the development of adventitial vasa vasorum is enhanced, extending from the stent implantation sites to the proximal and distal edges. Accumulated inflammatory cells (eg, M1-macrophages and mast cells) expressing inflammatory cytokines and vasoactive substances (eg, IL-1 $\beta$ ) at the adventitia of stent edges causes Rho-kinase activation. Subsequently, enhanced Rhokinase activity, particularly in medial vascular smooth muscle cells, leads to coronary hyperconstricting responses at the stent edges. Rho-kinase inhibitors (eg, hydroxyfasudil) suppress coronary hyperconstricting responses. DES, drug-eluting stents; EEL, external elastic lamina; IEL, internal elastic lamina.

site of inflammation. ${ }^{13,16,17,21,22} \mathrm{We}$ and others have previously examined the inflammatory changes only at the stent implantation sites. ${ }^{10,12,15,16}$ Of note, in the present study, we demonstrate that the adventitial inflammatory changes, including infiltration of pro-inflammatory M1-macrophages and mast cells and IL- $1 \beta$ expression, were significantly enhanced at the SES edge portions as compared with the BES edges. Importantly, there was a positive correlation between pro-inflammatory M1-macrophage density in the stent edges and the extent of coronary vasoconstriction in vivo. Furthermore, along with the decrease in adventitial inflammatory changes at 6 months after DES implantation, serotonin-induced coronary hyperconstricting responses also diminished. These results suggest that adventitial inflammatory changes at stent edges are strongly associated with the pathogenesis of DES-induced coronary hyperconstricting responses. Interestingly, it was previously demonstrated that mast cells are accumulated at the adventitia of the spastic coronary arteries of patients with variant angina, suggesting the important role of adventitial inflammation. ${ }^{31}$ We have previously demonstrated that Rho-kinase activation in the coronary artery is caused by chronic adventitial inflam-

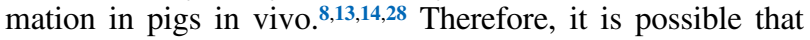
adventitial inflammation at the stent edges enhances activation of the Rho-kinase pathway at the stent edges after DES implantation. Furthermore, it has been demonstrated that inflammatory cytokines promote adventitial $\mathrm{VV}$ neovascularization, ${ }^{32}$ 
suggesting that inflammation could also be another therapeutic target to reduce both adventitial VV augmentation and coronary hyperconstriction after DES with the durable polymers implantation.

\section{DES-Induced Coronary Hyperconstriction and Rho-Kinase Activation}

Enhanced Rho-kinase activity plays a central role in the molecular mechanisms of coronary artery spasm. ${ }^{8,13,14,33}$ Rhokinase suppresses myosin phosphatase activity by phosphorylating MYPT1 and thus augmenting VSMC contraction. . $^{8,13,14}$ Serotonin induces significant hypercontractions in vitro and phosphorylation of the myosin-binding subunit of myosin phosphatase, a marker of Rho-kinase activation, at the IL- $1 \beta$ treated coronary spastic segments even after endothelium removal as compared with control coronary segments without endothelium, for which Y-27632, a specific Rho-kinase inhibitor, markedly inhibited. ${ }^{13}$ We have previously shown that intracoronary administration of fasudil or hydroxyfasudil, selective Rho-kinase inhibitors, inhibits coronary spasm in porcine models with various inflammatory stimuli $(\mathrm{eg}, \mathrm{IL}-1 \beta)$ at the adventitia in pigs in vivo, ${ }^{13}$ as well as in humans. ${ }^{8,14}$ We and others have demonstrated an inward progression of vascular inflammation from the adventitia toward the media and the intima. ${ }^{8,13,14,17,18}$ The present study demonstrates for the first time that in line with our previous findings at the stent implantation sites, ${ }^{15,16}$ the expression and activity of Rho-kinase at the stent edges are markedly enhanced, particularly in the adventitial inflammatory cells and the medial VSMC, and was significantly suppressed at the BES edges. Furthermore, serotonin-induced coronary vasoconstriction was equally abolished by hydroxyfasudil in the SES and BES sites. It is thus suggested that enhanced Rho-kinase activity in VSMC at SES edges, in response to adventitial inflammation, causes coronary hyperconstriction as compared with BES edges. This notion is supported by the present finding that positive correlations were noted between the extent of coronary vasoconstriction/M1-macrophage density and that of Rho-kinase activity at the stent edges.

\section{Study Limitations}

Several limitations should be mentioned for the present study. First, because we only used SES and BES in the present study, it remains to be elucidated which component of DES (eg, stent strut, polymer and anti-proliferative drug) is responsible for vascular inflammation. In addition, a first-generation SES is no longer commercially available at present. However, a SES has been deployed in millions of patients and thus efforts should be made to improve coronary vasomotion in those patients implanted with SES. Second, because the present study was performed in normal male pigs without pre-existing coronary atherosclerotic lesions, caution is required when extrapolating the present findings to humans. However, we were able to examine the pure foreign body reaction of DES by using healthy animals. Third, vascular healing processes in the present study with Göttingen miniature pigs, including neointimal formation and inflammation, were different from those in the previous study with Yukatan miniature pigs; ${ }^{34}$ this was probably due to differences in the extent of vascular injury and species of miniature pigs. ${ }^{35}$ Fourth, we used intracoronary serotonin to examine coronary vasoconstriction, as previously described. ${ }^{13-16}$ It is known that in porcine coronary arteries, acetylcholine does not cause endothelium-dependent relaxations due to the lack of cholinergic receptors on the endothelium. ${ }^{36}$ Fifth, because it was difficult to perform immunostainings of the resin-embedded sections of the stent implantation sites, we were unable to elucidate the detailed mechanism of VV neovascularization in the stent implantation sites. Sixth, the structural changes of adventitial VV before and after stent implantation in humans still remain to be elucidated. We have recently demonstrated that at 9-12 months after stent implantation, adventitial VV formation on the optical frequency domain imaging system ${ }^{37}$ is significantly enhanced at the stent edges of everolimus-eluting stents with durable polymers as compared with BES in humans in vivo. ${ }^{38}$ Finally, although we were able to demonstrate the association of the abnormalities in the adventitia (eg, inflammation, VV angiogenesis and Rhokinase activation), the detailed time-course and interactions among them remain to be examined in future studies.

\section{Conclusions}

The present study demonstrates that augmented VV formation and inflammatory changes in the adventitia are associated with the pathogenesis of DES-induced coronary hyperconstriction through the Rho-kinase pathway in pigs in vivo.

\section{Acknowledgments}

The authors wish to thank Y. Watanabe and A. Nishihara for their excellent technical assistance, and Asahi Kasei Pharma for providing hydroxyfasudil.

\section{Funding}

This work was supported, in part, by the grants-in-aid for the Scientific Research (18890018) and the Global COE Project (F02), and the grantsin-aid (H22-Shinkin-004) from the Japanese Ministry of Education, Culture, Sports, Science, and Technology, Tokyo, Japan (to H.S.) and the grant for young investigators of translational research from Tohoku University Hospital (to Y.M.).

\section{Conflicts of Interest}

H.S. is a consultant of Asahi Kasei Pharma.

\section{References}

1. Morice MC, Serruys PW, Sousa JE, Fajadet J, Ban Hayashi E, Perin $\mathrm{M}$, et al. A randomized comparison of a sirolimus-eluting stent with a standard stent for coronary revascularization. N Engl J Med 2002; 346: $1773-1780$.

2. Spaulding C, Daemen J, Boersma E, Cutlip DE, Serruys PW. A pooled analysis of data comparing sirolimus-eluting stents with baremetal stents. N Engl J Med 2007; 356: 989-997.

3. Nakazawa G, Finn AV, Joner M, Ladich E, Kutys R, Mont EK, et al. Delayed arterial healing and increased late stent thrombosis at culprit sites after drug-eluting stent placement for acute myocardial infarction patients: An autopsy study. Circulation 2008; 118: 1138-1145.

4. Takeda M, Shiba N, Takahashi J, Shimokawa H. A case report of very late stent thrombosis with peri-stent coronary artery aneurysm and stent-related coronary vasospasm. Cardiovasc Interv Ther 2013; 28: $272-278$.

5. Aizawa K, Yasuda S, Takahashi J, Takii T, Kikuchi Y, Tsuburaya R, et al. Involvement of Rho-kinase activation in the pathogenesis of coronary hyperconstricting responses induced by drug-eluting stents in patients with coronary artery disease. Circ J 2012; 76: 2552-2560.

6. Togni M, Windecker S, Cocchia R, Wenaweser P, Cook S, Billinger $\mathrm{M}$, et al. Sirolimus-eluting stents associated with paradoxic coronary vasoconstriction. J Am Coll Cardiol 2005; 46: 231-236.

7. Hamilos MI, Ostojic M, Beleslin B, Sagic D, Mangovski L, Stojkovic S, et al. Differential effects of drug-eluting stents on local endothelium-dependent coronary vasomotion. J Am Coll Cardiol 2008; 51: 2123-2129.

8. Shimokawa H. 2014 Williams Harvey Lecture: Importance of coronary vasomotion abnormalities-from bench to bedside. Eur Heart $J$ 2014; 35: 3180-3193.

9. Garg S, Bourantas C, Serruys PW. New concepts in the design of drug-eluting coronary stents. Nat Rev Cardiol 2013; 10: 248-260.

10. Tada N, Virmani R, Grant G, Bartlett L, Black A, Clavijo C, et al. 
Polymer-free biolimus A9-coated stent demonstrates more sustained intimal inhibition, improved healing, and reduced inflammation compared with a polymer-coated sirolimus-eluting Cypher stent in a porcine model. Circ Cardiovasc Interv 2010; 3: 174-183.

11. Stefanini GG, Kalesan B, Serruys PW, Heg D, Buszman P, Linke A, et al. Long-term clinical outcomes of biodegradable polymer biolimus-eluting stents versus durable polymer sirolimus-eluting stents in patients with coronary artery disease (LEADERS): 4 year follow-up of a randomised non-inferiority trial. Lancet 2011; 378: 1940-1948.

12. Pendyala LK, Matsumoto D, Shinke T, Iwasaki T, Sugimoto R, Hou $D$, et al. Nobori stent shows less vascular inflammation and early recovery of endothelial function compared with cypher stent. JACC Cardiovasc Interv 2012; 5: 436-444.

13. Kandabashi T, Shimokawa H, Miyata K, Kunihiro I, Kawano Y, Fukata Y, et al. Inhibition of myosin phosphatase by upregulated Rho-kinase plays a key role for coronary artery spasm in a porcine model with interleukin-1 $\beta$. Circulation 2000; 101: 1319-1323.

14. Shimokawa H, Takeshita A. Rho-kinase is an important therapeutic target in cardiovascular medicine. Arterioscler Thromb Vasc Biol 2005; 25: 1767-1775.

15. Shiroto T, Yasuda S, Tsuburaya R, Ito Y, Takahashi J, Ito K, et al Role of Rho-kinase in the pathogenesis of coronary hyperconstricting responses induced by drug-eluting stents in pigs in vivo. $J \mathrm{Am}$ Coll Cardiol 2009; 54: 2321-2329.

16. Tsuburaya R, Yasuda S, Shiroto T, Ito Y, Gao JY, Aizawa K, et al. Long-term treatment with nifedipine suppresses coronary hyperconstricting responses and inflammatory changes induced by paclitaxeleluting stent in pigs in vivo: Possible involvement of Rho-kinase pathway. Eur Heart J 2012; 33: 791-799.

17. Shimokawa H, Ito A, Fukumoto Y, Kadokami T, Nakaike R, Sakata $\mathrm{M}$, et al. Chronic treatment with interleukin- $1 \beta$ induces coronary intimal lesions and vasospastic responses in pigs in vivo: The role of platelet-derived growth factor. J Clin Invest 1996; 97: 769-776.

18. Maiellaro K, Taylor WR. The role of the adventitia in vascular inflammation. Cardiovasc Res 2007; 75: 640-648.

19. Wilson SH, Herrmann J, Lerman LO, Holmes DR Jr, Napoli C, Ritman EL, et al. Simvastatin preserves the structure of coronary adventitial vasa vasorum in experimental hypercholesterolemia independent of lipid lowering. Circulation 2002; 105: 415-418.

20. Kolodgie FD, Gold HK, Burke AP, Fowler DR, Kruth HS, Weber DK, et al. Intraplaque hemorrhage and progression of coronary atheroma. N Engl J Med 2003; 349: 2316-2325.

21. Drinane M, Mollmark J, Zagorchev L, Moodie K, Sun B, Hall A, et al. The antiangiogenic activity of $\mathrm{rPAI}-1_{23}$ inhibits vasa vasorum and growth of atherosclerotic plaque. Circ Res 2009; 104: 337-345.

22. Tanaka K, Nagata D, Hirata Y, Tabata Y, Nagai R, Sata M. Augmented angiogenesis in adventitia promotes growth of atherosclerotic plaque in apolipoprotein E-deficient mice. Atherosclerosis 2011; 215: 366-373.

23. Kwon HM, Sangiorgi G, Ritman EL, Lerman A, McKenna C, Virmani R, et al. Adventitial vasa vasorum in balloon-injured coronary arteries: Visualization and quantitation by a microscopic threedimensional computed tomography technique. J Am Coll Cardiol 1998; 32: 2072-2079.

24. Schwartz RS, Edelman ER, Carter A, Chronos N, Rogers C, Robinson KA, et al. Drug-eluting stents in preclinical studies: Recommended evaluation from a consensus group. Circulation 2002; 106: $1867-1873$.

25. Hirata Y, Tabata M, Kurobe H, Motoki T, Akaike M, Nishio C, et al. Coronary atherosclerosis is associated with macrophage polarization in epicardial adipose tissue. J Am Coll Cardiol 2011; 58: 248-255.

26. Maseri A, Beltrame JF, Shimokawa H. Role of coronary vasoconstriction in ischemic heart disease and search for novel therapeutic targets. Circ J 2009; 73: 394-403.

27. Virmani R, Kolodie FD, Farb A, Lafont A. Drug-eluting stents: Are human and animal studies comparable? Heart 2003; 89: 133-138.

28. Miyata K, Shimokawa H, Kandabashi T, Higo T, Morishige K, Eto $\mathrm{Y}$, et al. Rho-kinase is involved in macrophage-mediated formation of coronary vascular lesions in pigs in vivo. Arterioscler Thromb Vasc Biol 2000; 20: 2351-2358.

29. Sahler LG, Davis D, Saad WE, Patel NC, Lee DE, Waldman DL. Comparison of vasa vasorum after intravascular stent placement with sirolimis drug-eluting and bare metal stents. J Med Imaging Radiat Oncol 2008; 52: 570-575.

30. Herrmann J, Lerman LO, Rodriguez-Porcel M, Holmes DR Jr, Richardson DM, Ritman EL, et al. Coronary vasa vasorum neovascularization precedes epicardial endothelial dysfunction in experimental hypercholesterolemia. Cardiovasc Res 2001; 51: 762-766.

31. Forman MB, Oates JA, Robertson D, Robertson RM, Roberts LJ 2nd, Virmani R. Increased adventitial mast cells in a patient with coronary spasm. $N$ Engl J Med 1985; 313: 1138-1141.

32. Campbell KA, Lipinski MJ, Doran AC, Skaflen MD, Fuster V, McNamara CA. Lymphocytes and the adventitial immune response in atherosclerosis. Circ Res 2012; 110: 889-900.

33. Nihei T, Takahashi J, Tsuburaya R, Ito Y, Takagi Y, Matsumoto Y, et al. Circadian variation of Rho-kinase activity in circulating leukocytes of patients with vasospastic angina. Circ J 2014; 78: $1183-$ 1190.

34. Carter AJ, Aggarwal M, Kopia GA, Tio F, Tsao PS, Kolata R, et al. Long-term effects of polymer-based, slow-release, sirolimus-eluting stents in a porcine coronary model. Cardiovasc Res 2004; 63: 617-624.

35. Hiranuma N, Shinke T, Kakazawa G, Otake H, Matsumoto D, Ijichi $\mathrm{T}$, et al. Optial coherence tomography and histopathplogy assessment after implantation of first- and second-generation drug-eluting stents in a procine coronary model. Circ J 2014; 78: 2665-2673.

36. Graser T, Leisner H, Tiedt $\mathrm{N}$. Absence of role of endothelium in the response of isolated porcine coronary arteries to acetylcholine. Cardiovasc Res 1986; 20: 299-302.

37. Nishimiya K, Matsumoto Y, Takahashi J, Uzuka H, Odaka Y, Nihei $\mathrm{T}$, et al. In vivo visualization of adventitial vasa vasorum of the human coronary artery on optical frequency domain imaging: Validation study. Circ J 2014; 78: 2516-2518.

38. Nishimiya K, Matsumoto Y, Uzuka H, Oyama K, Tanaka A, Taruya $\mathrm{A}$, et al. Accuracy of optical frequency domain imaging for evaluation of coronary adventitial vasa vasorum formation after stent implantation in pigs and humans: A validation study. Circ J 2015; 79: $1323-1331$

Supplementary File 1

\section{Supplementary Files}

Methods

Table S1. Procedural parameters

Table S2. Histomorphometry at the stent implantation sites

Table S3. Histomorphometry at the stent edges

Table S4. Comparison between the proximal and distal stent edges

Figure S1. Coronary vasodilating responses.

Figure S2. Histology at the stent implantation sites.

Figure S3. Histological staining for various components in the stent edges of coronary adventitia.

Figure S4. Correlations between M2-macrophage density and coronary vasoconstricting responses/Rho-kinase activity.

Figure S5. Correlations between Rho-kinase activity and coronary vasoconstricting responses.

Figure S6. Correlations between M1-macrophage density and Rhokinase activity.

Please find supplementary file(s);

http://dx.doi.org/10.1253/circj.CJ-15-0149 